\title{
ARTIGOS
}

\section{ANÁLISIS DE LA IMPLICACIÓN: CONSTRUCCIÓN DEL SUJETO Y DEL OBJETO DE INVESTIGACIÓN}

MARÍA SOLEDAD MANRIQUE - MARÍA FLORENCIA DI MATTEO • LORENA SANCHEZ TROUSSEL

\section{RESUMEN}

Se toma como objeto de estudio un abordaje metodológico que permite al investigador trabajar en su implicación -la relación que establece con su propio objeto de conocimiento. Este abordaje metodológico se denomina análisis de la implicación. Se describe y analiza la forma en que el análisis de la implicación fue empleado en tres investigaciones educativas realizadas en Argentina en distintos momentos de la investigación. En un proceso de doble hermenéutica analizamos el procedimiento metodológico realizado oportunamente en cada estudio. El trabajo proporciona evidencia empírica sobre tres funciones diferentes del análisis de la implicación: la toma de decisiones, el distanciamiento sujeto-objeto y la construcción de conocimiento.

\section{MÉTODO DE INVESTIGACIÓN • INVESTIGACIÓN CUALITATIVA • ESTUDIO DE CASOS • INVESTIGACIÓN EDUCATIVA}

\section{INVOLVEMENT ANALYSIS: CONSTRUCTION OF THE RESEARCH SUBJECT AND OBJECT}

\section{ABSTRACT}

This article presents a methodological approach which allows the researcher to work on the relationship between subject and object in research. This approach is called involvement analysis. This paper describes and analyzes how this approach was used in three educational projects at different stages of the research, in Argentina. We have analyzed the methodological procedure in each research project using a double hermeneutic process. This paper provides empirical evidence on three different possible functions of analysis of involvement: decision making, establishing a distance from the research subject to the object and knowledge construction. 


\section{ANALYSE DE L'IMPLICATION: CONSTRUCTION DU SUJET ET DE L'OBJET DE LA RECHERCHE}

RÉSUMÉ

Cet article présente une approche méthodologique qui permet au chercheur de travailler sur son implication - la relation qu'il établit avec son propre objet de connaissance. Cette approche est connue sous le nom d'analyse de l'implication. La manière dont l'analyse de l'implication a été mobilisée dans trois recherches éducatives réalisées en Argentine à différents moments est décrite et analysée, Nous avons étudié les procedés méthodologiques mis en place dans chaque recherche à l'aide d'un processus de double herméneutique. L'article apporte des évidences empiriques sur trois fonctions différentes de l'analyse de l'implication: la prise de décision, la prise de distance du sujet-objet et la construction des connaissances.

MÉTHODE DE RECHERCHE - RECHERCHE QUALITATIVE • ÉTUDE DE CAS • RECHERCHE EN ÉDUCATION

\section{ANÁLISE DO ENVOLVIMENTO: CONSTRUÇÃO DO SUJEITO E DO OBJETO DE PESQUISA}

RESUMO

Este artigo tem como objeto de estudo uma abordagem metodológica que permite ao pesquisador refletir sobre seu envolvimento e a relação estabelecida com o seu próprio objeto de conhecimento. Essa abordagem é chamada de análise da implicação. Aqui, descrevemos e analisamos a forma como essa abordagem foi utilizada em três pesquisas educacionais na Argentina em diferentes momentos. Em um processo de dupla hermenêutica, analisamos o procedimento metodológico realizado em cada pesquisa. O artigo fornece evidência empírica sobre três diferentes funções possíveis de análise da implicação: a tomada de decisões, o distanciamento sujeito-objeto e a construção de conhecimentos. 


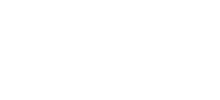

STE TRABAJO FOCALIZA EN LA RELACIÓN DEL SUJETO INVESTIGADOR CON SU OBJETO de estudio que, desde un enfoque particular de investigación cualitativa -el enfoque clínico (SOUTO, 2010)-, se denomina implicación. Más específicamente se analiza un abordaje metodológico que permite al investigador trabajar sobre su propia implicación. En la línea del enfoque clínico que abordamos, este tipo de trabajo se denomina análisis de la implicación (BARBIER, 1977; LOURAU, 1975; DEVEREUX, 1977). Mostraremos ciertos modos de hacer uso de este procedimiento en distintos momentos del proceso de investigación. El trabajo nos permitirá dar cuenta empíricamente de las funciones que cumple el análisis de la implicación tanto para la investigación cuanto para el investigador.

Tomamos como unidad de análisis tres investigaciones cualitativas diferentes del campo de la formación y la enseñanza en Argentina, realizadas cada una de ellas a través de un estudio de casos. Las tres se encuentran en fases distintas. Se llevó a cabo un trabajo de doble hermenéutica: un análisis (mediado por la escritura) sobre el análisis de la implicación realizado oportunamente en cada una de las investigaciones. Los tres estudios se enmarcan en el enfoque clínico entendido en sentido amplio (SOUTO, 2010). Este enfoque alude tanto a un posicionamiento epistemológico como a un abordaje metodológico. Postula que la construcción de conocimiento tiene lugar a partir del estudio en profundidad de la singularidad y no desde la identificación de 
regularidades. La singularidad incluye, asimismo, a un sujeto investigador también singular, cuya subjetividad participa de la construcción del objeto. Esta relación imbricada entre sujeto y objeto de la investigación se denomina implicación. En tanto ineludible la implicación debe ser puesta en análisis. El abordaje metodológico que propone llevar a cabo este análisis se denomina análisis de la implicación. Proporciona información tanto sobre el sujeto como sobre el objeto mismo, es decir, que contribuye con la construcción de conocimiento (DEVEREUX, 1977; FILLOUX, 1992; BLANCHARD-LAVILLE, 1996; BEILLEROT; BLANCHARD-LAVILLE; MOSCONI, 1996; DE GAULEJAC, 2002; TARACENA, 2002; CIFALI; GIUST-DESPRAIRIES, 2006).

Este trabajo se organiza de la siguiente manera. En primer lugar, presentamos perspectivas teórico-epistemológicas y metodológicas de diferentes autores provenientes de los campos sociológicos y antropológicos sobre la temática de la relación del sujeto investigador con su objeto de investigación en las Ciencias Sociales. Luego, exponemos las características del enfoque clínico y del análisis de la implicación como abordaje metodológico. Mostramos en los resultados tres formas que tomó el trabajo con la subjetividad del investigador durante distintas instancias del proceso de investigación en los tres estudios empíricos seleccionados. El análisis del abordaje metodológico llevado a cabo al interior de cada una de ellos nos permite sostener como hipótesis que el trabajo sobre sí mismo involucrado en el análisis de la implicación facilita la visibilización por parte del sujeto investigador de su propio posicionamiento con respecto al objeto de análisis. Esta visibilización, a su vez, cumplió tres funciones diferenciadas según las fases en que se encontraba cada una de las investigaciones: la reorientación en la toma de decisiones al inicio de la investigación, el distanciamiento y la distinción sujeto-objeto durante la recolección de datos y la construcción de conocimiento en la instancia de análisis de los datos.

\section{LA CENTRALIDAD DEL INVESTIGADOR Y SU SUBJETIVIDAD EN LA INVESTIGACIÓN CUALITATIVA}

Más allá de las diferentes tradiciones existentes en la investigación cualitativa, entendemos que ésta se caracteriza por una serie de prácticas interpretativas y materiales que estudian los fenómenos en los escenarios en que éstos transcurren, tratando de interpretarlos en función de los significados que las personas les otorgan (DENZIN; LINCOLN, 2011). Permanecer en los escenarios implica para un investigador establecer relaciones con su objeto de estudio. El registro de esa experiencia involucra, necesariamente, una dimensión subjetiva (DEVEREUX, 1977; ROCKWELL, 2011; GUBER, 2013). 
En la investigación de tradición positivista se dio prioridad a técnicas y métodos que se proponían excluir la subjetividad del investigador y garantizar la objetividad, situación ligada a la búsqueda de universalidad y generalización de los resultados. El uso acrítico de procedimientos y mediciones provenientes de esta tradición encontró duras críticas en la década de los ochenta (ROCKWELL, 2011).

A partir de estas críticas y del reconocimiento de la inevitabilidad de la presencia de la subjetividad-sentimientos, emociones, intuicionesdel investigador y de los demás actores, como rasgo propio de toda investigación social, desde distintas perspectivas epistemológicas y teóricas provenientes del campo de la sociología y la antropología, comenzó a plantearse la necesidad de considerarla como parte del proceso de investigación (DEVEREUX, 1977; ELIAS, 1990; SIRVENT, 1999; BOURDIEU, 2006; GALINDO, 2009; 2013; ROCKWELL, 2011; SOUTO, 2010; GUBER, 2013; MAZZA, 2015). Se proponen, con mayor o menor grado de detalle, pues, modalidades de abordaje de esta subjetividad en relación con el objeto de estudio, que es, a su vez, un sujeto social. A continuación presentamos algunas de estas perspectivas provenientes de diferentes áreas del conocimiento del campo de las Ciencias Sociales, que se inscriben en marcos teórico-epistemológicos que plantean formas de abordaje metodológico particulares.

Desde el campo de la etnografía, Guber (2013) señala la dificultad del encuentro con la alteridad, en tanto ésta desafía el sistema de clasificación y significación del analista. No se trata solamente de conceptos teóricos, sino que aquello que se pone a prueba son los propios patrones de pensamiento del analista. Investigador e informantes no sólo provienen de mundos socioculturales distintos, sino que también sostienen objetivos diferentes, lo cual genera contradicciones y malentendidos entre ambos. Partiendo de esta dificultad, Guber propone realizar un permanente control del investigador sobre sí mismo para reconocer y explicitar el origen de sus supuestos y de sus inferencias.

Desde el campo educativo, Sirvent (1999) propone un método de trabajo concreto con la subjetividad como parte de que lo que denomina historia natural de la investigación. Consiste en una narración o crónica que realiza el investigador durante las distintas etapas de la investigación. Permite hacer explícitas las decisiones adoptadas en el planteo original y en las sucesivas modificaciones del objeto de estudio, la recolección y el análisis de datos -entre otras- con el fin de analizar su racionalidad y consistencia e incrementar la objetivación de las conclusiones.

Galindo (2009, 2013), por su parte, desde una perspectiva sociológica y con el fin de revisar el modo en que se construyen los conocimientos científicos prestando atención tanto a los productos como a los procesos en la investigación, propone utilizar la biografía de investigación. Ésta es entendida como índice de aprendizaje estructural 
que ocurre tanto de manera consciente como inconsciente (en tanto aquello no reflexionado). La biografía se elabora a partir de un diario de investigación escrito regularmente para plasmar reflexiones sobre preocupaciones académicas y personales acerca del proceso subjetivo de volverse investigadores. Éstas pueden incluir problemas conceptuales y metodológicos relativos a la definición y cambios en el objeto de estudio, preferencias, sentimientos y preocupaciones durante el análisis, entre otros.

Elías (1990), en el marco de las tensiones dadas entre el compromiso (como implicación con el mundo social) y el distanciamiento del investigador con su objeto, analiza la construcción del conocimiento científico como un aspecto más dentro del proceso de civilización que se da al distanciarse y controlar las pasiones y emociones. El distanciamiento es definido como la capacidad del científico de diferenciarse emocionalmente (incluyendo la ideología) de los objetos de estudio, observarlos críticamente y alcanzar un conocimiento racional. Es evidente en este caso la intención de control de la subjetividad con el fin de alcanzar la objetividad.

En el marco del socioanálisis, especialmente desde la noción de objetivación participante, Bourdieu (2006), a partir de una crítica sobre la elaboración de diarios de reflexión u otras formas de explicitación de la "experiencia vivida del sujeto cognoscente" como insuficientes por quedar en el plano discursivo, postula la necesidad de reflexionar sobre las condiciones sociales de posibilidad de esas experiencias. Esta objetivación de la relación subjetiva con el objeto es necesaria, según él, para ejercer la vigilancia epistemológica en los distintos momentos de la investigación, creando así la condición para alcanzar la objetividad científica.

Al igual que en la perspectiva de Elías (1990) se aborda la dimensión subjetiva del investigador, no desde un punto psicológico singular, sino en tanto sujeto social. Bourdieu (2006) profundiza este aspecto añadiendo que ese sujeto social se posiciona, además, dentro de una comunidad académico-profesional. Se reflexiona, pues, sobre los presupuestos del investigador que informan sobre rasgos comunes del campo científico del cual forma parte. Esas características comunes remiten a tradiciones, hábitos de pensamiento, creencias, problemáticas, modos de actuar, rituales, valores implícitos; rasgos existentes en la estructura organizacional de la disciplina y en las propias categorías de conocimiento ya creadas.

Dentro del campo de la etnografía en educación, Rockwell (2011) suma a las miradas hasta aquí planteadas, la consideración de lo inconsciente en el vínculo investigador -objeto de investigación bajo la forma de defensas y ansiedades que aparecen en el proceso de investigación. Recomienda realizar un registro de las sensaciones vividas 
durante el trabajo de campo incluyendo, por ejemplo, el aburrimiento, el agotamiento, la fascinación o la curiosidad ante lo sucedido. Sin embargo, esta autora no desarrolla un método para abordar tal registro. Ello permite inferir que considera al registro como modalidad suficiente para el trabajo con la subjetividad, lo cual permite volver posteriormente a las notas con mayor distancia.

Los enfoques reseñados han sido recuperados por diversos investigadores en Latinoamérica para reflexionar sobre cómo los aspectos personales, subjetivos y profesionales se hacen presentes en la investigación (CABRERA, 2010; DI NAPOLI, 2014; CREGO, 2014).

En síntesis, los distintos autores referenciados sostienen que las características subjetivas del investigador se ponen de manifiesto en la vinculación, el abordaje y comprensión del objeto de estudio en el campo de las Ciencias Sociales. Guber (2013), Galindo (2009, 2013) y Elías (1990) consideran como condición importante reflexionar sobre los procesos personales y subjetivos en tanto aspectos conscientes e inconscientes -entendidos como no reflexionados- como sostienen Guber (2013) y Galindo (2009, 2013); o las pulsiones y pasiones a las que refiere Elías (1990) a fin de lograr un autoconocimiento (GALINDO, 2009), un distanciamiento y un conocimiento racional (ELIAS, 1990) sobre el proceso y los resultados de investigación (GALINDO, 2009, 2013; GUBER, 2013). Por su parte, Bourdieu (2006) y Rockwell (2011) complejizan la mirada al considerar aspectos inconscientes de la subjetividad del investigador. Mientras que el primero refiere a lo inconsciente colectivo, Rockwell remite a lo inconsciente subjetivo individual.

En todos los casos, el trabajo sobre la subjetividad que se propone como método provee cierta vigilancia sobre el proceso de investigación para garantizar el control de dicha subjetividad, el distanciamiento y separación entre el sujeto y el objeto de análisis. Mientras que algunos reconocen no saber aún cuál es el mejor método para lograr este distanciamiento (ELIAS, 1990), otros sugieren un trabajo sistemático de reflexión: ya sea a través de relatos de biografías (GALINDO, 2009, 2013), o de la historia natural de la investigación (SIRVENT, 1999; SARLE, 2003).

\section{EL TRABAJO SOBRE LA SUBJETIVIDAD DESDE EL ENFOQUE CLÍNICO}

Desde una tradición hermenéutica y comprensiva, el enfoque clínico se caracteriza por el abordaje en profundidad de la singularidad de cada situación encontrada o sujeto que se constituya en unidad de análisis, sin pretensión de generalizar o universalizar (BLANCHARD-LAVILLE, 2004; SOUTO, 2010; MAZZA, 2015). El enfoque clínico supone una epistemología: un modo de entender la relación entre el sujeto y el objeto, que implica asimismo una particular manera de investigar 
(DEVEREUX, 1977; FILLOUX, 1992; ARDOINO, 1993; BEILLEROT; BLANCHARD-LAVILLE; MOSCONI, 1996). Al igual que las líneas hasta aquí mencionadas se plantea la necesidad de considerar la dimensión de la subjetividad en investigación. Pero a diferencia de aquellas, desde el enfoque clínico la finalidad no es controlar esta subjetividad, sino poner esa subjetividad al servicio de la producción de conocimiento. Lo que se supone desde este enfoque es que sujeto y objeto están íntimamente ligados, por tanto, son indisociables (DEVEREUX, 1977; SOUTO, 2010; MAZZA, 2015). La propuesta en este caso es, entonces, utilizar esta ligazón para producir conocimiento, no intentar eludirla, separarla ni controlarla (DEVEREUX, 1977). Este trabajo sobre la subjetividad como abordaje metodológico involucrado en un planteo epistemológico cualitativo como lo es el enfoque clínico, lleva el nombre de análisis de la implicación. Quienes se alinean en esta perspectiva proponen una serie de dispositivos y recursos metodológicos para realizar este trabajo sobre uno mismo (FERRY, 2008), modalidades que no aparecen de manera explícita en los autores hasta ahora reseñados.

En esta línea el análisis es entendido como una búsqueda de sentidos que los actores otorgan a la situación: se trata de sentidos manifiestos, ocultos, inconscientes. En este proceso se construyen hipótesis interpretativas que pretenden dar cuenta de los rasgos singulares de las situaciones estudiadas. Con este fin interpretativo se toman múltiples teorías (ARDOINO, 1993) dentro de las cuales se incluyen las corrientes socioanalítica del análisis institucional y el psicoanálisis. Es posible establecer una distinción al interior del enfoque clínico entre las líneas que emplean el psicoanálisis de modo exclusivo como marco analítico -enfoque clínico en sentido estricto- de aquellas que incluyen el psicoanálisis junto con otras teorías-enfoque clínico en sentido amplio (SOUTO, 2010).

En el enfoque clínico, la implicación es entendida como el conjunto de relaciones, vínculo particular y absolutamente inevitable que entablan en el proceso de conocimiento, el investigador y el objeto de investigación, sea un sujeto individual, colectivo o social. Este vínculo puede incluir tanto dimensiones conscientes como inconscientes (BARBIER, 1977; LOURAU, 1975). Esta acepción del término implicación surge en el marco del análisis institucional (LOURAU, 1975) y refiere a los mecanismos psíquicos que llevan al propio analista/investigador a interpretar la realidad social, las acciones propias y de otros como si fueran transparentes y unívocas. Estos mecanismos escapan al control y voluntad del analista/investigador y por ello la implicación requiere ser analizada. Para Lourau (1975, 1993), el investigador/analista está implicado con su objeto de investigación/intervención, las instituciones de pertenencia y referencia, las demandas sociales, la epistemología del 
propio campo disciplinario y la escritura o cualquier otro medio que sirva para exponer los resultados de la investigación.

Savoye (HESS; SAVOYE, 1993), discípulo de Lourau, propone distinguir en el análisis de la implicación tres dimensiones: la organizacional/material, la ideológica/política y la libidinal/afectiva. De manera similar, Barbier (1977) propone tres dimensiones para pensar la implicación: estructuro-profesional; histórico-existencial y psicoafectiva. La primera de ellas refiere al vínculo que el investigador establece con su objeto en tanto se encuentra posicionado en el sistema de producción de acuerdo a su pertenencia socioeconómica y detenta valores provenientes de la misma. La dimensión histórico-existencial involucra el tipo de ligazón que se establece con sujetos reales y con una realidad vinculada con la actualidad del investigador y, nuevamente, en relación con sus valores como parte de una clase social determinada. La tercera dimensión expresa la confrontación con los aspectos más profundos de la personalidad del investigador, donde se ponen en juego relaciones transferenciales y contratransferenciales con el objeto de trabajar los temores y deseos y la posibilidad de elaborarlos.

Existe una serie de investigaciones recientes que podemos situar en la línea que propone el análisis de la implicación como modo de abordaje metodológico de la subjetividad. Esto puede ser empleado tanto en la investigación (BLANCHARD-LAVILLE, 2004; CLOT; LEPLAT, 2005; SOUTO, 2010; ANDREUCCI, 2012; MAZZA, 2015), como en la intervención en la formación profesional de tipo clínica (BLANCHARD-LAVILLE, 2004, 2013; CLOT; LEPLAT, 2005; ANDREUCCI, 2012).

Blanchard-Laville $(2004,2013)$ lleva a cabo dispositivos de carácter clínico con docentes, inspirados en los grupos Balint desde hace 20 años. El objetivo de estos grupos de trabajo es la función profesionalizante, es decir, lograr una modificación profunda y con efecto duradero en el funcionamiento profesional. La forma de trabajo consiste en analizar la implicación personal en los actos profesionales a través de un dispositivo basado en el intercambio oral entre los integrantes del grupo, acerca de un relato de una situación real de uno de ellos. El grupo elabora hipótesis que le permite a quien relata la situación, revisar su modo inicial de interpretarla. Se enfatiza, allí, la necesidad de cuidar, especialmente, la cualidad analítica de los intercambios y evitar juicios evaluativos sobre el narrador. Este dispositivo permite a los docentes relacionar el nivel personal con el rol profesional, posibilitando la elaboración psíquica de los conflictos - una metabolización de las emociones, más que una comprensión intelectual o de representaciones conscientes-, la cual deriva en una mejor capacidad para pensar de los participantes (BION, 1980). Esta metabolización de las emociones levanta las prohibiciones de sentir y enseña a retomar contacto con lo experimentado subjetivamente para poder elaborarlo. 
En la misma línea, Cifali y Giust-Desprairies (2006) sostienen que una mirada clínica debe estar acompañada de una ética de la acción. Ésta implica conocer y escuchar al otro, ponerse en su lugar y, al mismo tiempo, escucharse a sí mismo desde las resonancias con el otro, sosteniendo las incoherencias, las contradicciones y la opacidad de lo que termina develándose (ARDOINO, 1997). Para lograrlo proponen dispositivos de reflexión que permiten analizar las representaciones psíquicas y sociales de la formación y las teorías implícitas del docente a través de talleres de escritura, de expresión, de diarios de formación o bitácoras, relatos de prácticas o de foros electrónicos de discusión.

Andreucci (2012), por su parte, propone el desarrollo de lo que denomina el “ojo pedagógico". Éste es entendido como la capacidad de analizar la propia implicación-las creencias y sentimientos-al interior de las situaciones de enseñanza, como modo de apuntalar las dimensiones socioafectivas y éticas de los profesores y prevenir alteraciones en la trama relacional que afectan su desempeño, calidad de vida y bienestar físico-emocional. Para ello, propone un trabajo mediante el método de estudio de casos con docentes. Busca desarrollar el "ojo pedagógico”, habilidad que implica, para la autora, focalizar la atención con el fin de identificar un problema, pero también diversificar la atención para considerar dos o más fuentes de información a la vez, elaborar hipótesis comprensivas sobre las situaciones con precisión y exactitud cognitiva y afectivo-sensitiva, hipótesis que serán, luego, contrastadas en las situaciones concretas y revisadas. El "ojo pedagógico” se constituye en una destreza adquirible -una unión de lo artístico-intuitivo y lo científico-racional- que permite hacer un uso práctico del conocimiento implícito, tácito o inconsciente sobre la enseñanza del otro y el autoconocimiento personal, trayéndolo a la conciencia (SCHON, 1994; BLANCHARD-LAVILLE, 2004, 2013; CIFALI; GIUST-DESPRAIRIES, 2006).

Hemos señalado diferentes usos del análisis de la implicación en situaciones de formación profesional. Este método también puede ser empleado en investigación. En ese caso se trata de poner en análisis las notas que el investigador consignó sobre cómo lo afectaron las situaciones vividas en instancias de recolección y análisis de datos. Se sugiere tomar estas notas sobre sensaciones, impresiones, pensamientos y creencias propios de la subjetividad de quien observa, en una columna paralela al registro completo de la situación, a la que llamamos "tercera columna". El uso de esta columna permite el registro simultáneo a la observación de cómo lo observado afecta al observador, qué le suscita, qué le hace pensar y sentir. De este modo dicha información no se pierde y se ubica en un espacio físico diferenciado. Estos datos registrados contribuyen a la construcción del objeto de investigación, y son retomados luego y discutidos con un otro, ya sea el director del proyecto o el equipo de investigación. En el presente trabajo, para analizar la implicación de 
las investigadoras se pondrán en análisis estos registros en la tercera columna.

Es posible realizar el análisis de la implicación en los distintos momentos del proceso investigativo. Inicialmente, permite hacer visible el tipo de vinculación que cada investigador establece con la situación y los sujetos en su entrada a terreno. Se trata de un trabajo que permite acceder al juego de relaciones transferenciales y contratransferenciales que se dan entre el sujeto investigador y el sujeto investigado (MAZZA, 2015).

Una segunda instancia de análisis de la implicación tiene lugar en el momento de trabajo con los datos. Una nueva relación se establece allí entre el analista y aquello que se manifiesta de la situación a través del dato construido (SOUTO, 2010; MANRIQUE; SOUTO; TENAGLIA, 2014; MAZZA, 2015). En este caso, el análisis apunta a dilucidar qué dice del objeto de investigación aquello que el investigador ha experimentado subjetivamente. Nuevamente, se torna fundamental la presencia de un tercero para mediar la interpretación de este material.

Como hemos planteado hasta aquí, el enfoque clínico tiene en cuenta la subjetividad del investigador, pero de un modo diferente a como lo hacen los enfoques etnográficos. Su especificidad radica en la inclusión de la dimensión inconsciente en dicha subjetividad, lo cual requiere un tratamiento particular. Incorpora, por tanto, técnicas y categorías de marcos psicoanalíticos (FILLOUX, 1992; BEILLEROT; BLANCHARD-LAVILLE; MOSCONI, 1996; CIFALI; GIUST-DESPRAIRIES, 2006).

\section{METODOLOGÍA}

Con el objetivo de analizar el modo en que se vincula el investigador con su objeto de análisis y de identificar el aporte de esta metodología a la tarea del investigador y a la producción de conocimiento, se consideraron tres investigaciones en el ámbito educativo argentino enmarcadas en el enfoque clínico en sentido amplio (SOUTO, 2010). Cada una de ellas trabaja, metodológicamente, con estudio de casos. Las tres investigaciones tienen sede de trabajo en el Instituto de Investigaciones en Ciencias de la Educación de la Facultad de Filosofía y Letras de la Universidad de Buenos Aires. Las dos primeras tienen como fuente de financiamiento al Consejo Nacional de Investigaciones Científicas y Técnicas; la tercera a la Secretaría de Ciencia y Técnica de la Universidad de Buenos Aires.

El criterio de selección de esta muestra es coherente con el objetivo de mostrar las funciones que cumple el empleo de este procedimiento en el proceso de investigación en su totalidad. Por ello se buscaron investigaciones que estuvieran en diferentes etapas: una de ellas en la fase previa a la toma de datos, otra durante la recolección 
de datos y una tercera posterior a la toma de datos, cuando ya se están realizando los análisis finales.

La primera investigación, situada en su etapa previa a la recolección, tuvo como objetivo estudiar la evaluación de los aprendizajes en el marco de instancias de formación pre profesional en cursos universitarios y analizar en profundidad las vinculaciones con el proceso formativo del sujeto y su relación con características de la profesión. Se trabajó con cuatro estudios de caso de universidades nacionales. En este artículo se comunica la situación acontecida en un momento inicial del proceso de trabajo de campo: la búsqueda de un caso.

El segundo de los proyectos, que se encontraba en la etapa de recolección de datos, se proponía describir y analizar la experiencia de directivos que trabajan en escuelas primarias en contextos de vulnerabilidad social, atendiendo a los tipos de dificultades que enfrentan en el ejercicio del cargo, así como las capacidades y competencias que ponen en juego para su resolución. La unidad de análisis fueron directores de escuelas primarias de gestión pública que reciben a población de barrios urbano-marginados.

La tercera investigación considerada, que se encuentra en una etapa de análisis de los datos, tuvo por objeto analizar la formación docente en profesorados de enseñanza primaria, en especial en lo que refiere al campo de la formación en las prácticas y dentro de ella a la residencia, es decir, las prácticas en el aula. Se tomó como unidad de análisis una docente a la que se acompañó durante el proceso.

\section{PROCEDIMIENTO DE RECOLECCIÓN Y ANÁLISIS DE DATOS}

Tomamos como unidad de análisis de las tres investigaciones dos fuentes de datos:

a) Las anotaciones en la tercera columna de los registros escritos que confeccionamos durante la recolección de datos mientras observamos las situaciones como investigadores en el campo, siguiendo la metodología antes descripta (SOUTO, 2010; MAZZA, 2015).

b) Las elaboraciones subsiguientes que se hicieron de ese material (MAZZA, 2015) volviendo sobre esas anotaciones de modo reflexivo a partir de diferentes consignas y con el acompañamiento de cada directora de la investigación. El producto del proceso fue volcado a la misma columna.

Diferenciamos dos momentos del trabajo metodológico sobre el análisis de la implicación: a lo largo del proceso de recolección de los datos y durante el análisis.

Se procedió de la siguiente manera: 
a) Se tomaron notas en una tercera columna durante la recolección y análisis de los datos según la metodología ya explicada (SOUTO, 2010; MAZZA, 2015).

b) Se trabajó con las notas tomadas en esta columna siguiendo las siguientes consignas:

- Leer las propias notas y responder: ¿qué siento yo en relación con las cosas que he consignado en los datos"? Para esto primero tratar de describir "desde afuera" los estados afectivos que se observan en uno mismo. Ver cuáles predominan.

- Preguntarse luego: ¿qué dice de mi posicionamiento frente al objeto toda esta descripción de estados que atravesé? ¿Con quién me estoy identificando/enfrentando? ¿Dónde me estoy ubicando como observadora de la situación?

- Por último, ¿hasta qué punto eso que siento -mi propio modo de estar afectado-puede incidir en la lectura que estoy haciendo de las situaciones vividas tanto al comienzo como durante la toma de datos?

- En el caso particular de la última investigación, además de estas preguntas, se sumaron las siguientes:

- ¿Los estados afectivos vivenciados sufrieron algún cambio a lo largo del proceso?

- ¿Es posible establecer una relación entre los estados afectivos vividos $y$ los hechos consignados?

- ¿Qué de lo que me afecta en relación con los datos refiere a mí mismo y qué refiere al objeto?

- ¿En qué momentos lo que refiere a mí mismo y lo que refiere al objeto se diferencian?

- Cuando lo que me afecta refiere al objeto, ¿qué dice de él? Es decir: ¿qué de la propia implicación nos informa sobre el objeto mismo?

Para analizar el procedimiento, se categorizó el material antes descripto (las anotaciones y respuestas a las preguntas) a través del análisis de contenido (KRIPPENDORF, 1990), considerando como dimensiones los tipos de implicación antes mencionados: estructuro-profesional; histórico-existencial y psicoafectivo (BARBIER, 1977).

Para dar cuenta de la forma que tomaron estas dimensiones de la implicación en cada una de las tres investigaciones nos valimos de conceptos provenientes de la teoría psicoanalítica tales como idealización, proyección, pantalla, tolerancia a la frustración (FREUD, 1915; KLEIN, 1971; BION, 1980) y análisis institucional (LOURAU, 1975; VARELA, 2004).

\section{RESULTADOS}

Mostraremos en cada una de las investigaciones cuál fue el análisis realizado sobre la implicación dando cuenta de diferentes usos posibles de este 
abordaje metodológico. Se emplea la primera persona del singular en tanto cada análisis ha sido llevado a cabo por el propio sujeto sobre sí.

\section{SOBRE EL CONTACTO INICIAL, LA LLEGADA Y LA TOMA DE DECISIONES A PARTIR DEL ANÁLISIS DE LA IMPLICACIÓN (INVESTIGACIÓN 1)}

Con motivo de realizar el contacto inicial con una docente a cargo de una cátedra de una universidad pública, cuyas prácticas de enseñanza y evaluación me interesaba tomar como objeto de estudio, envié un correo electrónico y la visité. Inicialmente la profesora se mostró -desde lo discursivo- en apariencia predispuesta para mi incorporación en ese espacio de formación con fines de investigación. Sin embargo, se generaron diversas circunstancias que me permitieron comprender, análisis de la implicación mediante, que el trabajo de investigación no sería allí posible. Entre aquellas situaciones, desde un punto de vista descriptivo, ubicamos: la demanda de permisos ante autoridades de la universidad que realicé sin obtener respuesta alguna, un pedido de entrevista que fue respondido solicitando los tópicos de la indagación, un ofrecimiento de un espacio y tiempo horario acotado para realizarla $\mathrm{y}$, por último, el requisito de un listado con tipo y cantidad de actividades de trabajo de campo y tiempo estimado para cada una de ellas.

$\mathrm{Al}$ interpretar estas circunstancias pude resignificar la situación y considerar estos sucesos descriptos como modos confusos en las comunicaciones. En un momento posterior reinterpreté estos sentidos atribuidos por mí a la situación como indicios de cierta resistencia por parte de la profesora. A pesar de esto, continué insistiendo en tomar los datos hasta tanto pude poner en análisis mi propia implicación que presento a continuación.

Desde el punto de vista de la implicación psicoafectiva, pude reconocer un inicio con deseos y altas expectativas por tomar el caso y una frustración inicial por no poder hacerlo. Sentía temor de no poder acceder a un caso que, en mi imaginario, cumplía con todos los requisitos necesarios. El análisis de la implicación me permitió problematizar por qué yo pensaba que el caso tenía todos los rasgos deseados por mí y logré darme cuenta de que ese ideal era solo un supuesto previo. Por otra parte, pude relacionar mi insistencia en continuar con el caso a pesar de las resistencias de la profesora, con un rasgo particular propio: mi perseverancia y al mismo tiempo mi intolerancia al límite que me planteaba el otro, en este caso el sujeto de la investigación.

El trabajo con mi directora me habilitó la posibilidad de formular nuevas preguntas para construir sentidos e hipotetizar acerca del lugar que yo estaría ocupando en el imaginario de esta docente y los efectos que esto podría tener en su modo de actuar. A partir de la pregunta de qué era lo que me pasaba a mí con mi objeto de estudio -otro sujeto-y qué le pasaba a él conmigo, percibo inicialmente una evitación como 
modo de rechazo por parte de la directora; situación que me genera incomodidad, temor por no poder iniciar el trabajo de campo allí y una reacción de insistencia de mi parte para poder lograrlo.

Al tomar distancia de esta emoción, pude analizar nuevamente los sucesos y construir otras interpretaciones sobre ese rechazo. En ese otorgar sentido surge tanto una imagen que yo creo haber proyectado en ella como otras que yo imagino que ella se habría formado sobre mí. Estas reinterpretaciones me permitieron pensar que el rechazo de la docente se ligaba a ciertos temores que podrían relacionarse con el lugar que representaría yo en su imaginario en varios sentidos. En primer lugar, mi tema de investigación -la evaluación de los aprendizajespodría situarme a sus ojos como una posible evaluadora, algo que probablemente yo pensaría de otros en una situación similar. En segundo lugar, esos temores se podrían dar al verme como investigadora de la Universidad y del campo de las Ciencias de la Educación, lugar investido de saber sobre la enseñanza. Y en relación con todo ello, interpreté que la docente podría verme como una amenaza en tanto alguien capaz de develar tensiones ocultas de su posición en su propia institución. Incluso podríamos pensar que yo misma hubiera contribuido a este sentido, con mis proyecciones sobre ella. Finalmente, una tercera lectura mía se ligó a una interpretación que realicé acerca de la vaguedad de la noción de la profesora sobre la investigación en el campo de la educación. Al no conocer este campo, ella podría no comprender mi rol y podría estar imaginando situaciones que generaron el rechazo asociado a ese temor.

Desde el análisis de la implicación en una dimensión histórico-existencial consideré especialmente aportes teóricos provenientes de una perspectiva institucional. Desde allí entendí que las prácticas de enseñanza situadas en cátedras universitarias son en sí mismas instituciones que presentan un cerco institucional (LOURAU, 1975) que opera diferenciando simbólicamente un interior de un exterior. Si esa cerrazón garantiza la integridad (VARELA, 2004), la apertura a una investigación implicaría que un otro ajeno (investigador o equipo) hiciera públicas las imperfecciones que suceden al interior de ese espacio cerrado. Su inclusión podría generar en los miembros -en este caso, la docente-, temores y ansiedades inconscientes.

Desde la dimensión estructuro-profesional de la implicación, pude pensar que el rechazo por parte de la docente a que tomara la cátedra para la investigación me generaba un conflicto valorativo en el sentido de que considero que ella debería haber abierto su espacio de trabajo para la investigación, ya que la construcción del conocimiento es una de las principales funciones de la universidad. Pensé, además, que esta tarea debería estar facilitada por pertenecer ambas a la misma universidad. En este aspecto me identifiqué negativamente con la profesora ya que yo misma, como docente de la universidad, abriría el espacio de mi aula para 
la investigación. Esperaba que la profesora respondiera con reciprocidad, desde "un código moral de servicio" (BARBIER, 1977). Las preguntas de análisis de la implicación sobre aquello que la situación dice de mí como sujeto y qué dice sobre el objeto permitió comprender rasgos de ese objeto: darle un sentido al rechazo de la docente y comprender mi perseverancia para realizar en esa cátedra el trabajo de campo.

El análisis de la implicación desde las dimensiones psicoafectiva, histórico-existencial y estructuro-profesional, en el momento de ingreso al campo, brindó aportes para entender, por un lado, mi deseo y ansiedad por tomar el caso, el temor y la frustración por no acceder a él, junto con la insistencia y la dificultad inicial para tolerar ese rechazo. En todo este proceso, se tornó necesaria la relación con un otro real, en este caso, la directora del proyecto, quien actuó como continente (BLANCHARD-LAVILLE, 2004), ayudando a tomar esas emociones y devolverlas modificadas, facilitando que yo pudiera tolerar la frustración, sublimar esos deseos en pensamiento y tomar la decisión de buscar un nuevo caso para la investigación.

El análisis de la implicación hizo luz sobre una situación compleja que se presentaba de modo ambiguo y al hacerlo cumplió la importante función de contribuir con la toma de decisiones al interior de un proyecto de investigación.

\section{SOBRE EL DIFERENCIAR SUJETO-OBJETO DURANTE LA TOMA DE DATOS (INVESTIGACIÓN 2)}

En el caso de la investigación sobre directores, en tanto el objetivo era comprender los rasgos que asume la experiencia de dirigir una escuela, para la reconstrucción de dicha experiencia la permanencia en el campo se tornaba fundamental. En términos metodológicos se tomaron entrevistas al director del caso y se realizaron observaciones de sus jornadas de trabajo. Esto significó acompañar al director en sus diferentes actividades, durante varias horas y a lo largo de varios meses.

Tomaremos aquí para el análisis de la implicación, las impresiones registradas en dos momentos precisos de ese proceso de recolección de datos: la entrevista inicial al director y la observación de una jornada de trabajo con los docentes de la escuela.

Transcribo a continuación parte del registro de impresiones: por un lado las impresiones registradas en el momento mismo de la entrevista; en segundo lugar, las impresiones registradas una vez terminada la misma.

Registro de impresiones durante la entrevista: "El director me resulta muy agradable."; "Me hace sentir cómoda, genera un espacio de cercanía, de trato poco protocolar."; "Lo que dice respecto del trabajo con las familias, con el barrio suena muy interesante, ojalá todos los directores pensaran así”; “el modo de entender la relación con el barrio 
es interesante, parece una postura muy democrática y comprometida”; "hay humildad, una idea de correrse y dejar que los otros hagan".

Registro de impresiones luego de la entrevista:

\begin{abstract}
La entrevista fue interesante. Me sentí cómoda y sentí que el director habló cómodamente. Me dio la impresión de ser una persona abierta, con ganas de trabajar y de producir cambios en la escuela y también en el barrio. Habló de manera sencilla, pero siendo ordenado en las ideas y utilizando categorías y conceptos que le permitían claridad en la presentación. Esto me sorprendió gratamente, quizás porque en la experiencia que tuve en cursos de directivos, la apertura hacia el barrio está menos presente, así como lo están determinadas lecturas teóricas. Termino con la ideasensación de que serían deseables más personas así en los cargos directivos. Ojalá pueda ver cómo se hace todo lo que él dice en la escuela.
\end{abstract}

El análisis de las primeras impresiones me permitió elaborar algunas hipótesis acerca de las motivaciones y deseos que me llevaron a realizar estudios en contexto de vulnerabilidad social.

En primer lugar, desde la dimensión psicoafectiva de la implicación puedo señalar que construí del director y su tarea una imagen altamente positiva. La misma se sostenía fundamentalmente en el trabajo que se proponía realizar con las familias de la escuela. Es decir, que valoré y focalicé mi mirada, desde el inicio, en el compromiso del director con las familias del barrio, y esto significaba para mí un director que se ocupaba de los sectores desfavorecidos de la sociedad. El director estaba preocupado por incorporar a las familias en la vida institucional de la escuela, así como por mejorar, en la medida de sus posibilidades, las condiciones de vida de las mismas. Se trataba de hombres y mujeres desocupados o con trabajos muy precarios, que encontraban dificultades para acceder a un trabajo digno, a atención de la salud, a una vivienda, etc.

Desde el punto de vista del aspecto histórico-existencial de la implicación, la mirada que construí sobre el director y su modo de trabajar con la población de la escuela se vincula con mi pertenencia de clase: soy, en mi familia, la primera en concurrir a la universidad y provengo de una familia de obreros. El hecho de focalizar mi mirada en su trabajo con los sectores populares puede interpretarse desde esta pertenencia.

La imagen que había construido (de manera más o menos consciente) del director, era la de un hombre comprometido con su tarea, que desarrollaba prácticas democráticas, de escucha y preocupación hacia los demás. Sin duda, esta imagen se apoyaba en lo que el director decía y también hacía. Pero de alguna manera, mi implicación histórico-existencial operó para que idealizara su figura (la viera sin contradicciones 
ni fisuras) y solo pude ver este proceso de idealización unos meses después, cuando observé una jornada de trabajo del director con los maestros de la escuela. Algunas de las impresiones registradas después de esa observación:

\begin{abstract}
Me voy con una sensación "amarga", siento cierta molestia con el director y cierta identificación con los docentes. Cosa que no me había pasado hasta ahora. Habló mucho, mucho, sin posibilidad alguna de escuchar lo que estaban diciendo los docentes. Me voy como enojada, desilusionada.
\end{abstract}

No es posible cambiar una escuela si no se escucha las preocupaciones de los docentes y se trabaja con ellos.

Me sentía enojada cuando salí de la reunión. Sentí frustración en ese momento y me costó bastante volver a la escuela después de ese encuentro.

El registro de las impresiones y la detección de la "sensación de enojo”, casi de "traición”, me permitió plantear algunos interrogantes: ¿por qué sentía enojo?, ¿por qué se me presentaba la idea de “traición”?, ¿qué me molestaba? Esta interrogación me abrió la puerta para pensar dos cuestiones. Por una parte, desde el aspecto estructuro-profesional de la implicación podía afirmar que no compartía el modo de trabajo del director con los docentes. Lo que allí había sucedido contradecía el modo en que yo consideraba que se debía trabajar con los maestros y me llevó a identificarme con ellos, cosa que no había sucedido hasta el momento.

Sin embargo, la consideración de los aspectos estructuro-profesionales no me parecía suficiente para dar cuenta de las emociones que había experimentado. Tuve que revisar las notas y solo después de ciertos señalamientos de mi directora de tesis, logré comprender que el enojo y la sensación de "traición” que sentía se fundaban en que estaba fuertemente desilusionada. Había construido del director una imagen sin fisura a raíz de cuánto había valorado su trabajo con las familias. El enojo que sentí luego de la observación de la jornada con los maestros seguramente tuvo que ver con que esa imagen construida se desarmó en ese encuentro. Pichon-Rivière (2010), en su teoría del vínculo, señala que el encuentro con el otro siempre tiene efectos subjetivos, en la medida en que se toma a ese otro como modelo o ideal, auxiliar o semejante, objeto de amor y/o deseo y como rival o enemigo.

La posibilidad de comprender este pasaje, este cambio abrupto en la mirada, como el de una zona que va de la ilusión a la desilusión, de la idealización a la desidealización, me permitió un acercamiento a los datos de otro tipo. Luego de este proceso, volví a la escuela y empecé a poder ver 
y registrar aspectos de la experiencia directiva, así como mirar los registros ya construidos desde otro lugar, que esa idealización no me había permitido considerar. El desafío, en el proceso de análisis de los datos, fue entonces para mí el de una vigilancia que me permitiera evitar lecturas dicotómicas que condujeran a una imagen del director como totalmente "bueno" o totalmente "malo" y poder interpretar la complejidad de la experiencia directiva con sus matices, contradicciones y ambigüedades.

En la pregunta acerca de qué de mi implicación refiere a mí misma y qué de ella refiere al objeto, aparece en este caso la posibilidad de conectarse, tomar conciencia y registrar la complejidad en el objeto que implica la coexistencia de aspectos que podrían ser considerados "buenos" y "malos" y la posibilidad de diferenciar uno mismo como sujeto investigador, de ese objeto.

\section{SOBRE LA CONSTRUCCIÓN DE CONOCIMIENTO A PARTIR DEL ANÁLISIS DE LA IMPLICACIÓN (INVESTIGACIÓN 3)}

En la investigación en la que compartí con una residente docente seis jornadas de su residencia, el trabajo de análisis realizado con acompañamiento de mi directora sobre las anotaciones en la tercera columna me permitió echar luz sobre aspectos referidos a la residente -el “objeto" de investigación, aspectos de mi propia subjetividad, aspectos comunes a ambas (que se complementaban o eran similares) y otros que se diferenciaban.

Desde la dimensión psicoafectiva, lo primero que reconozco en el análisis es que mi implicación se produce a partir de mi identificación con Francisca -la residente- como docente y como persona, en su angustia y miedo ante la necesidad de cumplir con lo que interpreta como demandas contradictorias ante la situación de ser evaluada. Al atender a las demandas externas en mayor medida que a los propios valores ella no consigue tomar una posición acorde a su criterio y sostenerla con decisiones, tal como yo siento que me ha pasado en muchas situaciones laborales. Por otra parte, me identifico con sus alumnos (y no con ella) en las instancias en las que percibo abuso de poder o autoritarismo.

Aquellos aspectos en donde me identifiqué me permitieron comprenderla mejor, juzgarla menos y abordar con mayor tolerancia los aspectos que me generaban rechazo -su autoritarismo, su labilidad.

Desde la dimensión estructuro-profesional pude despejar mi identificación negativa con Francisca. Habiendo trabajado como docente durante tantos años, en muchos momentos me encontré comparando mi quehacer en aquella época con lo que Francisca hacía, y criticando sus decisiones desde allí. Se ponía en juego sobre todo en actividades por las que yo tenía predilección, como la lectura de cuentos. Allí se me jugó claramente mi saber especializado sobre el tema. Mis propios valores y conocimientos contradicen lo que Francisca priorizaba en su práctica. En este caso el análisis de la implicación me permitió darme 
cuenta de que estaba ocupando una posición evaluativa al observar a Francisca. Este trabajo elaborativo realizado con el acompañamiento de mi directora, implicó la toma de conciencia y me ayudó a ubicarme nuevamente en un posicionamiento de investigadora, desde el cual pierde sentido la evaluación. Esta elaboración, a su vez, debe haber contribuido a que la empatía inicial con Francisca no se desarticulara y que continuara firme la confianza que ella había depositado en mí -aspectos intersubjetivos clave para poder recolectar datos en una investigación de estas características.

Desde la dimensión histórico-existencial lo que apareció en el análisis de mi implicación fue una fuerte identificación con los alumnos con quienes se cometían injusticias. Mi propia vivencia de abuso de poder en la infancia me ha hecho especialmente sensible a estas cuestiones y me permitió captar por identificación con ellos, muy rápidamente, situaciones de abuso o de maltrato que están naturalizadas en la escuela. La sutileza en este caso no hizo que pasaran desapercibidas para mí. Mi propia subjetividad me servía de filtro que no dejaba pasar estas pequeñas situaciones de niños a quienes se les negó la palabra o el protagonismo. Puso de manifiesto así, el autoritarismo de la residente, las propias contradicciones entre su discurso y lo que podía hacer en cada situación con los niños. Resulta evidente en este caso el modo en que el análisis de la implicación contribuye con la construcción de hipótesis interpretativas.

Fue la dimensión psicoafectiva la que aportó mayor riqueza al análisis de los datos. Fue significativo que después de las vacaciones de invierno dejé de tener ganas de ir a la escuela. Esta sensación de rechazo por mi parte, asociada a cierto desgano y pérdida de interés en observar a Francisca, al ser analizada, permitió terminar de darle una forma a la hipótesis acerca del abandono por parte de ella de su propio deseo, en favor del mandato de aprobar la evaluación de la residencia. A partir entonces de la confrontación entre ciertos comportamientos, los registros de lo dicho por Francisca y lo experimentado como sensación del investigador y consignado en tercera columna, elaboramos pues la hipótesis según la cual señalamos que la experiencia de la residencia para Francisca habría tomado el sentido de una ficción, de un requisito formal, de un pasaje de pruebas, algo que no deja huellas porque no es vivido como "real".

La ausencia de deseo en Francisca y su proyección de la apatía en mí habrían funcionado para acentuar el rechazo que sentí contratransferencialmente (FREUD, 1915). La sensación de que la experiencia podía pasar sin dejar huellas, colaboró también con la formulación de la hipótesis.

El análisis del rechazo observado en principio en mí nos permitió, pues, saber algo más del sujeto observado, de Francisca. El 
funcionamiento psíquico de la observadora -mío- funcionó en este caso a modo de espejo, reflejando de modo deformado algunos de los rasgos del objeto-sujeto, de Francisca.

Lo ocurrido en este caso nos permite mostrar cómo el análisis de la implicación contribuye con la construcción de conocimientos durante el proceso de análisis de los datos.

\section{DISCUSIÓN Y REFLEXIONES FINALES}

La descripción de las elaboraciones a las que dio lugar el análisis de la implicación en los tres casos considerados nos remite a las siguientes temáticas: las funciones que cumple el análisis de la implicación en los diferentes momentos de la investigación, la diferencia con otros modos de abordar la subjetividad, las diferentes dimensiones involucradas en la implicación de un analista -en coincidencia con la teoría de Barbier (1977)-, la relevancia de la presencia de un tercero en este tipo de trabajo subjetivo y las cualidades de este vínculo. Por último, otro tema que se pone de manifiesto desde los análisis de la implicación realizados fue de índole epistemológica: la relación de la investigación con la transformación subjetiva dentro de este paradigma. A continuación, desplegaremos cada una de estas cuestiones.

En primer lugar, el trabajo puso de manifiesto que el análisis de la implicación afecta la investigación de diferentes modos. En este sentido mostramos evidencia empírica sobre la conceptualización teórica desarrollada por Mazza (2015) acerca de las diferentes funciones que el análisis de la implicación cumple en las distintas etapas de la investigación. En este sentido la puesta en visibilidad del propio posicionamiento del investigador en relación a su objeto de estudio, que el análisis de la implicación favoreció, permitió en el caso 1 contribuir con la toma de decisiones al interior del proyecto advirtiendo la dificultad para establecer un contrato adecuado que hiciera posible la investigación. En el caso 2 permitió distinguir aspectos propios del analista de otros que provenían del objeto analizado. El caso 3 mostró un último aporte que nos brinda el análisis de la implicación: contribuir con la construcción de conocimiento, tomando como datos a ser analizados los aspectos que habían sido proyectados sobre el analista.

Las primeras dos funciones del análisis de la implicación (tomar decisiones y diferenciar sujeto-objeto) se asemejan a las posibilidades que ofrecen otros modos de trabajo con la subjetividad, por ejemplo desde el planteo de Bourdieu (2006) a través de la objetivación participante. No obstante, desde el marco metodológico del enfoque clínico, tal como se puso de manifiesto en los resultados del análisis de las investigaciones 1 y 2 , estas dos funciones se desempeñan de un modo cualitativamente 
diferente en tanto se incluyen aspectos inconscientes individuales en el análisis.

Por otra parte, los resultados también mostraron que el análisis de la implicación permite diferenciar sujeto de objeto. En este sentido nos parece relevante resaltar que esta diferenciación no implica de ninguna manera negar la ligazón entre ambos. No se produce una separación entre sujeto y objeto, sino que se admite su ineludible unión, aunque admitiendo que es posible a partir de una operación del pensamiento y de elaboración psíquica, establecer diferencias entre uno y otro.

La posibilidad de construir conocimiento a través del análisis sobre el investigador mismo, que constituye la tercera función del análisis de la implicación que mostramos en el caso 3, es exclusiva de este método. Es decir que el análisis de la implicación como método va más allá de los otros métodos propuestos en tanto en lugar de intentar controlar la implicación, se vale de ella y la utiliza a favor de la investigación. En línea con el planteo de Devereux (1977), nuestro trabajo muestra que, efectivamente, la subjetividad del investigador puede ser tratada como un objeto más a ser analizado, y que tal tratamiento arroja luz sobre el objeto, que en este caso era otro sujeto.

En esta línea el empleo de la teoría psicoanalítica como herramienta de análisis permite, precisamente, hacer interpretaciones acerca de la subjetividad y los vínculos establecidos, aspectos que quedan invisibilizados o que resultan inabordables desde otras teorías. Los conceptos de transferencia y proyección (FREUD, 1915) permiten pensar al analista como pantalla que, de ser analizada, permitiría abordar indirectamente el propio objeto, proporcionando información clave para la investigación. Estos conceptos fundamentan este abordaje, constituyendo a la subjetividad del analista como un instrumento más al servicio del análisis (DEVEREUX, 1977). Este modo de trabajo es el mismo que se ha empleado en otros casos aunque en ellos no se explicite (ARDOINO, 1993; MANRIQUE, SOUTO y TENAGLIA, 2014; MAZZA, 2015).

Por otro lado, al poner de manifiesto la relación entre el análisis de la implicación y la temporalidad, en relación con la etapa de la investigación en que se lleva a cabo, este trabajo muestra la necesidad de analizar la implicación a lo largo de todo el proceso de investigación, no sólo al comienzo del acercamiento al campo y durante la recolección de datos.

El trabajo también nos permitió comprobar que los tipos de implicación que Barbier (1977) propone -estructuro-profesional, histórico existencial y psicoafectiva- efectivamente constituyen una herramienta heurística útil a la hora de organizar el tipo de vínculo que un investigador establece con su objeto de estudio en diferentes 
momentos de la investigación. Pudimos, de hecho, categorizar nuestro material valiéndonos de estas dimensiones.

Por último, el trabajo también mostró que el análisis de la implicación llevado a cabo tuvo siempre como condición el lugar de un tercero, que fue ocupado en cada uno de los proyectos citados por la directora. Ahora bien, el hecho de que efectivamente este análisis en profundidad haya sido posible da cuenta del carácter de este vínculo entre investigador y director. Tomando el aporte de Winnicott $(1969,1986)$ podríamos caracterizar este vínculo como un espacio "suficientemente bueno”, en el que tuvo lugar una relación continente-contenido (BION, 1980) de crecimiento mutuo, donde el juicio de valor quedó suspendido. En este espacio quien ocupó el lugar de tercero, al poner en juego su capacidad de rêverie (BLANCHARD-LAVILLE, 2004) -de alojar al otro sin juzgarlo- hizo posible un retorno sobre sí mismo del investigador desde una distancia que permitió a la vez subjetivación y objetivación. Ahora bien, este trabajo de elaboración psíquica no sólo contribuyó con la investigación y la construcción de conocimientos sino también dio lugar a un crecimiento personal intrasubjetivo del investigador, es decir a su formación (FERRY, 2008).

En el marco de esta forma de hacer ciencia que desde lo epistemológico supone la inseparabilidad entre sujeto y objeto, todo investigador se encontrará permanentemente en proceso de formación, entendida como desarrollo de capacidades, habilidades y actitudes (FERRY, 2008; BARBIER, 1990). En tanto investigar implica llevar a cabo un trabajo de elaboración psíquica sobre uno mismo que es transformador, investigación y formación constituyen dos partes de un mismo fenómeno. Es esa subjetividad en permanente proceso de trans-formación la que es capaz de construir nuevos objetos o nuevos modos de mirarlos, al ser atravesada y afectada por ellos: sujeto y objeto construyéndose en un proceso indisociable.

\section{BIBLIOGRAFÍA}

ANDREUCCI, Paola M. El enfoque clínico en formación de profesores. Revista Profesorado, v. 16, n. 1, p. 257-275, 2012. Disponível em: <http://www.ugr.es/local/recfpro/rev161COL3.pdf>. Acesso em: Mar. 2015.

ARDOINO, Jacques. L'approche multiréférentielle (plurielle) des situations éducatives et formatives. Revue pratiques de formation: Analyses, Paris, n. 25-26, p. 1-14, 1993.

ARDOINO, Jacques. La implicación. In: CONFERENCIA IMPARTIDA EN EL CENTRO DE ESTUDIOS SOBRE LA UNIVERSIDAD. 4 de noviembre de 1997, México: UNAM, 1997.

BARBIER, Jean Marie. Prácticas de formación: evaluación y análisis. Buenos Aires: Novedades Educativas, 1990.

BARBIER, René. La recherche-action dans l'institution éducative. Paris: Gauthiers Villars, 1977. 
BION, Wilfred. Aprendiendo de la experiencia. Buenos Aires: Paidós, 1980.

BLANCHARD-LAVILLE, Claudine. Saber y relación pedagógica. Buenos Aires: Novedades Educativas, 2004.

BLANCHARD-LAVILLE, Claudine. Para una clínica grupal del trabajo docente. Revista del IICE, Buenos Aires, n. 34, p. 7-28, 2013.

BOURDIEU, Pierre. La objetivación participante. Apuntes de investigación del CECYP, n. 10, p. $87-101,2006$.

CABRERA, Paula. Volver a los caminos andados. Nuevas Tendencias en Antropología, n. 1, p. 54-88, 2010 .

CIFALI, Mireille; GIUST-DESPRAIRIES, Florence. De la clinique. Un engagement pour la formation et la recherche. Bruselas: De Boeck, 2006.

CLOT, Yves; LEPLAT, J. La méthode clinique en ergonomie et en psychologie du travail. Le Travail Humain, v. 4, n. 68, p. 289-316. 2005.

CREGO, María Laura. Estar en el campo: el investigador como sujeto y objeto de investigación. Revista Trabajo y Sociedad, n. 23, p. 343-352, 2014.

DE GAULEJAC, Vicent. Lo irreductible social y lo irreductible psíquico. Perfiles latinoamericanos, n. 21, p. 49-71, Dic. 2002.

DENZIN, Norman; LINCOLN, Yvonna. El campo de la investigación cualitativa. Barcelona: Gedisa. 2011.

DEVEREUX, George. De la ansiedad al método en las ciencias del comportamiento. México: Siglo XXI, 1977.

DI NAPOLI, Pablo. La objetivación participante en el trabajo de campo. Revista Nuevas Tendencias en Antropología, n. 5, p. 77-97. 2014.

ELÍAS, Norbert. Compromiso y distanciamiento. Ensayos de sociología del conocimiento.

Barcelona: Ediciones Península, 1990.

FERRY, Giles. Pedagogía de la formación. Buenos Aires: Noveduc, Facultad de Filosofía y Letras, Universidad de Buenos Aires, 2008.

FILLOUX, Jean Claude. La psychanalyse peut elle contribuer a la formation des enseignants? Practiques de formation, n. 23, p. 25-29, 1992.

FREUD, Sigmund. L'inconscient. In: FREUD, Sigmund. Metapsychologie. Paris: Gallimard, 1985. cap 1, p. 11-43.

GALINDO, Fernando. Volviéndose investigador en un contexto intercultural: Las paradojas de la "nueva" artesanía intelectual. Revista Trabajo y Sociedad, Santiago del Estero, v. 12, n. 13, p. 1-11, dic. 2009.

GALINDO, Fernando. La historia interna: la biografía de una tesis doctoral cualitativa. Trabajo y Sociedad, Santiago del Estero, n. 20, v. 17, p. 21-24, verano 2013.

GUBER, Rosana. El salvaje metropolitano. Reconstrucción del conocimiento social en el trabajo de campo. Buenos Aires: Paidós, 2013.

HESS, Remi; SAVOYE, Antoine. L'analyse institutionnelle. Qué-sais je? Paris: PUF, 1993.

KLEIN, Melanie. Contribuciones al psicoanálisis. Buenos Aires: Hormé, 1971.

KRIPPENDORF, Klaus. Método de análisis de contenido. Teoría y Práctica. Buenos Aires: Paidós, 1990.

LOURAU, René. El análisis institucional. Buenos Aires: Amorrortu, 1975.

LOURAU, René. René Lourau na UERJ. Análise institucional e práticas de pesquisa. Rio de Janeiro: UERJ, 1993.

MANRIQUE, María Soledad; SOUTO, Marta; TENAGLIA, Guadalupe. Aportes del Enfoque clínico a un estudio sobre residencias docentes en Argentina. In: INTERNATIONAL CONGRESS OF QUALITATIVE INQUIRY. 2014, Córdoba, Argentina. Revista de Ciencia y Técnica. Disponível em: $<$ http://www.21.edu.ar/investigacion-ponencias-congreso-investigacion-cualitativa-cienciassociales.html>. Acesso em: ago. 2015. 
MAZZA, Diana. El proceso de construcción de sentido en un enfoque clínico en sentido amplio. Educación, Lenguaje y Sociedad, v. XI, n. 11, p. 93-113, 2015.

PICHON-RIVIERE, Ernesto. Teoría del vínculo. Buenos Aires: Nueva Visión, 2010.

ROCKWELL, Elsie. La experiencia etnográfica. Historia y cultura de los procesos educativos. Buenos Aires: Paidós, 2011.

SARLE, Patricia. La historia natural en la investigación cualitativa. Revista del Instituto de Investigaciones en Ciencias de la Educación, v. IX, n. 21, p. 25-30, 2003.

SCHON, Donald. La formación de profesionales reflexivos. Hacia un nuevo diseño de la enseñanza y el aprendizaje en las profesiones. Barcelona: Paidós, 1994.

SIRVENT, María Teresa. Cultura popular y participación social. Una investigación en el barrio de Mataderos (Buenos Aires). Buenos Aires: Miño y Dávila, 1999.

SOUTO, Marta. La investigación clínica en ciencias de la educación. Revista del Instituto de Investigaciones en Ciencias de la Educación, v. XVII, n. 29, p. 57-74, 2010.

TARACENA, Elvia. La construcción del relato de implicación en las trayectorias profesionales. Perfiles Latinoamericanos, n. 21, p. 117-141, Diciembre 2002.

VARELA, Cristian. La entrada al terreno institucional. Tramas, México, n. 21, p. 219-237, 2004.

WINNICOTT, Donald W. Objets transitionnels et phenomenes transitionnels. 1951. In: WINNICOTT, Donald W. De la pediatrie a la psychanalyse. Paris: Payot, 1969. p. 169-186.

WINNICOTT, Donald W. Realidad y juego. Buenos Aires: Gedisa, 1986.

MARÍA SOLEDAD MANRIQUE

Instituto de Investigaciones en Ciencias de la Educación, Universidad de Buenos Aires/Consejo Nacional de Investigaciones Científicas y Técnicas de la República Argentina -CONICET-, Buenos Aires, Argentina

solemanrique@yahoo.com.ar

MARÍA FLORENCIA DI MATTEO

Instituto de Investigaciones en Ciencias de la Educación, Universidad de Buenos Aires /Consejo Nacional de Investigaciones Científicas y Técnicas de la República Argentina -CONICET-, Buenos Aires, Argentina

florenciadm@hotmail.com

LORENA SANCHEZ TROUSSEL

Instituto de Investigaciones en Ciencias de la Educación, Universidad de Buenos Aires /Consejo Nacional de Investigaciones Científicas y Técnicas de la República Argentina -CONICET-, Buenos Aires, Argentina lorenalbs@hotmail.com 